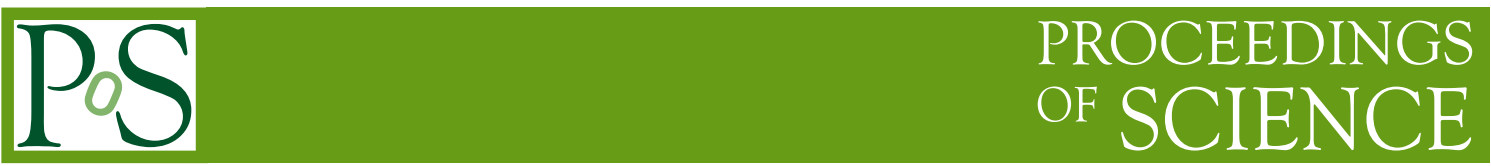

\title{
Overview of the latest jet physics results from ALICE
}

\author{
James Mulligan for the ALICE Collaboration ${ }^{a, b}$ \\ ${ }^{a}$ Nuclear Science Division, Lawrence Berkeley National Laboratory, Berkeley, California 94720, USA \\ ${ }^{b}$ Physics Department, University of California, Berkeley, CA 94720, USA \\ E-mail: james.mulligan@berkeley.edu
}

\begin{abstract}
We overview recent jet measurements in pp and $\mathrm{Pb}-\mathrm{Pb}$ collisions with the ALICE detector. ALICE reconstructs jets at midrapidity using the anti- $k_{\mathrm{T}}$ algorithm, including both charged particle jets from the ALICE tracking detectors, and full jets from the combination of the ALICE electromagnetic calorimeter with the tracking system. We focus on inclusive and semi-inclusive jet measurements as well as jet substructure measurements, including a variety of groomed and ungroomed observables. In pp collisions, these results test $\mathrm{pQCD}$ formalisms at $p_{\mathrm{T}}$, jet $<140 \mathrm{GeV} / c$ in a low pileup environment, and can constrain models of non-perturbative effects. In $\mathrm{Pb}-\mathrm{Pb}$ collisions, these measurements test models of jet quenching in the quark-gluon plasma, and can be used to constrain the properties of high temperature QCD matter.
\end{abstract}

40th International Conference on High Energy physics - ICHEP2020

July 28 - August 6, 2020

Prague, Czech Republic (virtual meeting) 


\section{Introduction}

Jet measurements can be used to study fundamental aspects of QCD in both pp and $\mathrm{Pb}-\mathrm{Pb}$ collisions. In pp collisions, jet measurements test perturbative calculations, which is important for our first-principles understanding of QCD, and inform which observables are under sufficient theoretical control to serve as suitable baselines for heavy-ion measurements. Jet measurements in pp collisions also constrain nonperturbative effects, such as hadronization. In $\mathrm{Pb}-\mathrm{Pb}$ collisions, jets serve as probes of the quark-gluon plasma (QGP). Comparing jet measurements in $\mathrm{Pb}-\mathrm{Pb}$ collisions to those in pp collisions allows us to investigate the modifications of jets due to their interaction with the QGP. By comparing models to data, we can constrain medium bulk properties such as transport coefficients, and potentially elucidate the nature of the degrees of freedom of the QGP.

In what follows, we highlight a selection of recent results from the ALICE experiment, with an emphasis on inclusive, semi-inclusive, and jet substructure measurements. We reconstruct jets at midrapidity using the anti- $k_{\mathrm{T}}$ algorithm with resolution parameters ranging from $R=0.1$ to $R=0.6$. These include both "charged jets" clustered only from charged particles, as well as "full jets" clustered with both charged and neutral particles. All presented results are corrected for detector effects (in both $\mathrm{pp}$ and $\mathrm{Pb}-\mathrm{Pb}$ collisions) and background fluctuations (in $\mathrm{Pb}-\mathrm{Pb}$ collisions).

\section{Jet measurements in proton-proton collisions}

\subsection{Inclusive cross-sections}

Inclusive jet measurements at low- $p_{\mathrm{T}}$ as a function of $R$ provide tests of the perturbative and nonperturbative (NP) contributions to the inclusive jet cross-section [1, 2]. First-principles calculations of the perturbative cross-section were recently computed at NLO with resummation of large logarithms [3-5], and to NNLO at fixed order [6,7]. The significance of various terms of the perturbative expansion, as well as the significance of the hadronization and underlying event (UE) effects, are important questions for our fundamental understanding of QCD.

ALICE recently reported inclusive jet cross-sections for jet resolution parameters $R=0.1-0.6$ over the range $20<p_{\mathrm{T}}$, jet $<140 \mathrm{GeV} / c$, shown in Fig. 1 (left) [1]. By covering a large range of $R$ down to low $p_{\mathrm{T}}$, these measurements span a range of perturbative regimes including small$R$ resummation, and a wide range of NP effects (from hadronization-dominated at small $R$ to UE-dominated at large $R$ ), which can be used to further constrain NP effects in pp collisions. Figure 1 (right) shows a comparison of these measurements to NNLO calculations [6] as well as to POWHEG+PYTHIA8 $[8,9]$. These predictions are consistent with the data for all $R$ and $p_{\mathrm{T}}$, jet, and along with other comparisons [1] demonstrate the importance of NNLO effects and NLL resummations. We additionally reported jet cross-section ratios of different $R$, which allow one to elucidate higher-precision effects of the $R$-dependence of the inclusive jet cross-section.

\subsection{Dynamical grooming}

Jet grooming techniques are used to reduce non-perturbative effects by selectively removing soft large-angle radiation, which allows for well-controlled comparisons of measurements to pQCD calculations [10-16]. The Dynamical grooming algorithm [12, 17] identifies a single "splitting" by re-clustering the constituents of a jet with the Cambridge-Aachen algorithm [18], and traversing 

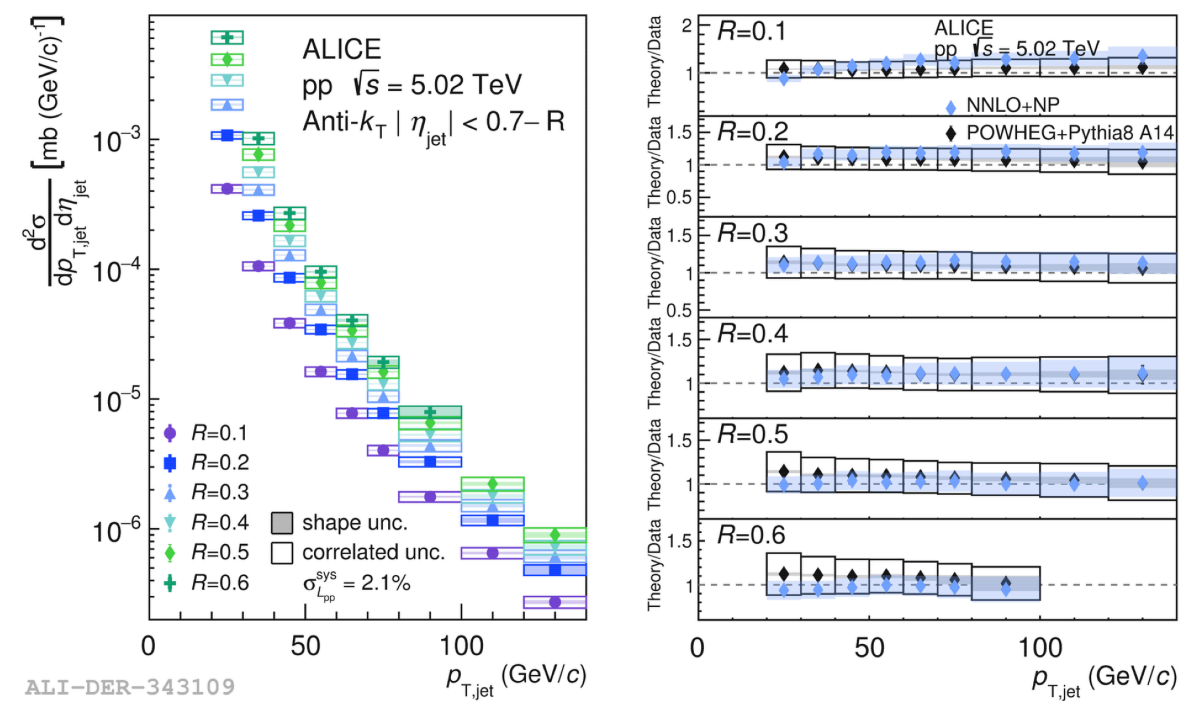

Figure 1: Measurements of inclusive jet cross-sections in pp collisions for $R=0.1-0.6$ (left) [1] and comparison to NNLO calculations [6] (with NP corrections) and POWHEG+PYTHIA8 (right) $[8,9]$.

the primary Lund plane [19] to identify the splitting that maximizes: $z_{i}\left(1-z_{i}\right) p_{\mathrm{T}, \mathrm{i}}\left(\frac{\Delta R_{i}}{R}\right)^{a}$, where $z_{i}$ is the longitudinal momentum fraction of the $i^{\text {th }}$ splitting, $\Delta R_{i}$ is the rapidity-azimuth $(y, \varphi)$ separation of the daughters, and $a$ is a continuous free parameter. Since the grooming condition defines a maximum rather than an explicit cut, every jet returns a tagged splitting. We focus on the two kinematic observables that characterize the splitting: the groomed jet radius, $\theta_{\mathrm{g}} \equiv R_{\mathrm{g}} / R \equiv$ $\sqrt{\Delta y^{2}+\Delta \varphi^{2}} / R$, and the groomed momentum fraction, $z_{\mathrm{g}} \equiv p_{\mathrm{T} \text {,subleading }} /\left(p_{\mathrm{T} \text {, leading }}+p_{\mathrm{T} \text {, subleading }}\right)$. Figure 2 (left) shows the $\theta_{\mathrm{g}}$ distributions in pp collisions for several values of the grooming parameter $a$. For small $a$, the grooming condition favors splittings with symmetric longitudinal momentum, reflected in the distributions skewing towards small $\theta_{\mathrm{g}}$. As $a$ increases, the grooming condition favors splittings with large angular separation, reflected in the distributions skewing towards large$\theta_{\mathrm{g}}$. The results are compared to PYTHIA [9], which describes the data well.

\subsection{Ungroomed jet angularities}

The class of infrared and collinear safe jet angularities [20] is defined as

$$
\lambda_{\beta}^{\kappa}=\sum_{i \in \mathrm{jet}}\left(\frac{p_{\mathrm{T}, i}}{p_{\mathrm{T}, \mathrm{jet}}}\right)^{\kappa}\left(\frac{\Delta R_{i}}{R}\right)^{\beta}
$$

for $\kappa=1$ and $\beta>0$. Jet angularities provide a flexible way to study QCD in both pp and $\mathrm{Pb}-\mathrm{Pb}$ $[15,21,22]$ collisions due to the ability to systematically vary the observable definition in a way that is theoretically calculable. Additionally, jet angularities give sensitivity to the predicted scaling of non-perturbative shape functions [23, 24]. Figure 2 (right) shows the $\lambda_{\beta}^{\kappa=1}$ distributions in pp collisions (right) for several values of $\beta$. As $\beta$ increases, the distributions skew towards small $\lambda_{\beta}^{\kappa=1}$, since $\Delta R_{i} / R$ is smaller than unity. The distributions become broader for smaller $R$ (not shown here), as expected due to the collinear nature of jet fragmentation. The results are compared to PYTHIA [9], which describes the data reasonably well. 

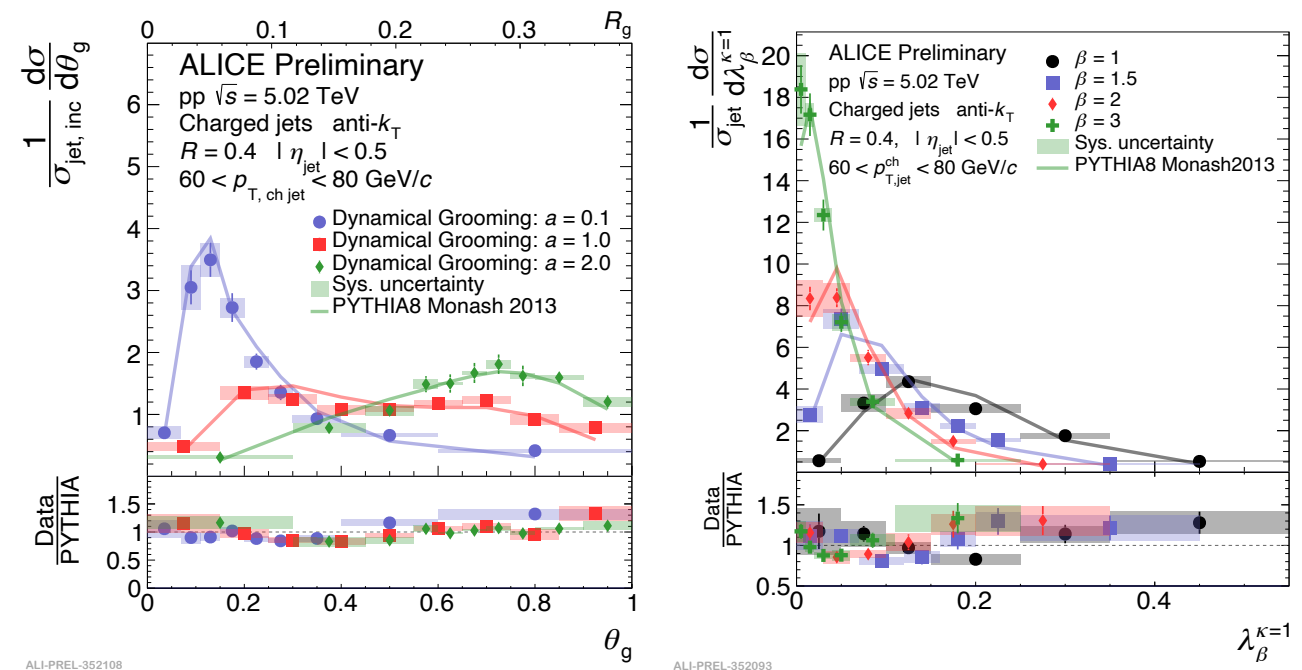

Figure 2: Measurements of $\theta_{\mathrm{g}}$ (left) in pp collisions with Dynamical Grooming [12] for three values of the grooming parameter $a$. Measurements of jet angularities (right) $\lambda_{\beta}^{k=1}$ in pp collisions with $R=0.4$ for four values of the continuous parameter $\beta$. Both results are compared to PYTHIA Monash 2013 [9].

\section{Jet measurements in heavy-ion collisions}

\subsection{Inclusive jet $R_{\mathrm{AA}}$}

The jet $p_{\mathrm{T}}$ spectrum in heavy-ion collisions is suppressed relative to that in (appropriately scaled) pp collisions, indicating that jets transfer energy to the hot QCD medium (see e.g. [1]). Extending measurements to low $p_{\mathrm{T}}$ and large $R$ is of particular interest, in order to constrain competing effects between the recovery of out-of-cone radiation and changes in the jet population (see e.g. [25]). To do this, ALICE has started exploring the use of machine learning (ML) to estimate the background-subtracted jet $p_{\mathrm{T}}$ on a jet-by-jet basis, reducing UE fluctuations at the expense of introducing model bias. Figure 3 (left) shows an example in which the ML method extends the inclusive jet measurement to lower $p_{\mathrm{T}}$ compared to traditional techniques [26]. The ML method is trained using PYTHIA, however, and is based on subtracting more $p_{\mathrm{T}}$ from jets with a higher fraction of soft particles (typically arising from the UE) relative to jets with a higher fraction of hard particles (typically arising from jet fragmentation). This method therefore assumes that jet modification in the QGP (which is known to induce soft and wide-angle energy flow) does not significantly alter the ML performance, otherwise the method will lead to uncontrolled errors in the $p_{\mathrm{T}}$ reconstruction. Several simple models of modified fragmentation are shown in Fig. 3 (left), and show significant model-dependence. These effects remain under investigation.

\subsection{Semi-inclusive hadron-jet correlations}

Semi-inclusive hadron-jet correlations are well-suited to statistical background subtraction procedures in heavy-ion collisions, which allows jet measurements to low $p_{\mathrm{T}}$ and large $R$ [28, 29]. By measuring the azimuthal angular separation between trigger hadrons and associated recoiling jets, one can test for large-angle jet deflection in the QGP [30] as well as transverse broadening (e.g. [31]). Figure 3 (right) shows the distribution of per-trigger semi-inclusive yields as a function of 

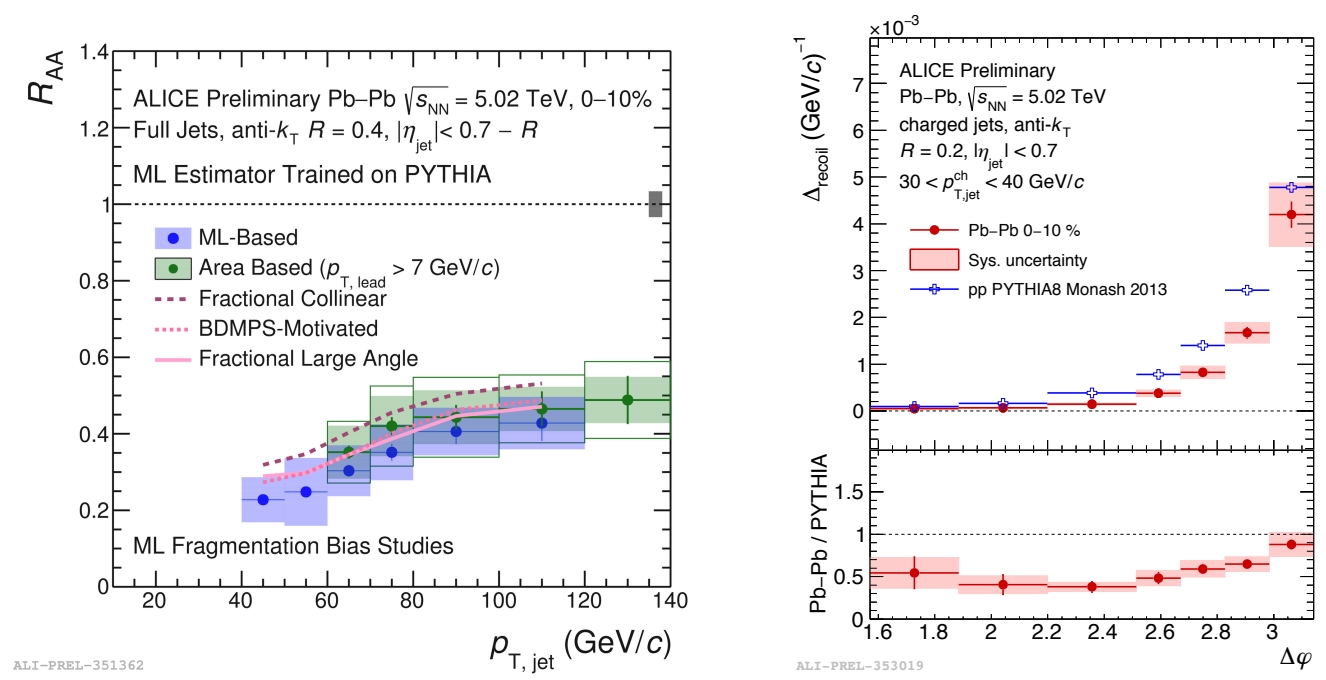

Figure 3: Measurements of jet $R_{\mathrm{AA}}$ (left) using a PYTHIA-based machine learning method for background subtraction [26], and per-trigger semi-inclusive jet yields (right) as a function of the azimuthal angle difference $\Delta \varphi$ between trigger hadrons and associated recoiling jets [27].

the azimuthal angle difference $\Delta \varphi$ between trigger hadrons and associated recoiling jets [27]. This is the first hadron-jet $\Delta \varphi$ distribution that is fully corrected for detector and background effects. The measurement shows an overall suppression of yields in $\mathrm{Pb}-\mathrm{Pb}$ collisions relative to PYTHIA, which is typical of medium-induced energy loss. The measurement also shows a relative narrowing of the $\Delta \varphi$ distribution towards $\Delta \varphi=\pi$. The origin of this apparent narrowing is unknown (see for example [32]), and demands further theoretical and experimental study.

\subsection{Soft Drop grooming}

Jet grooming techniques have been applied to heavy-ion collisions in order to explore whether jet quenching in the quark-gluon plasma modifies the hard substructure of jets [33-43]. The large UE poses a challenge, however, since fluctuations in the UE can cause groomed splittings to be misidentified [44]. Figure 4 shows measurements of the groomed momentum fraction, $z_{\mathrm{g}}$, and the groomed jet radius, $\theta_{\mathrm{g}}$, with the Soft Drop grooming algorithm [45]. By using stronger grooming conditions than previous measurements, the result has been fully corrected for detector effects and background fluctuations. We find that the $z_{\mathrm{g}}$ distributions in $\mathrm{Pb}-\mathrm{Pb}$ collisions are consistent with those in pp collisions, whereas a significant narrowing of the $\theta_{\mathrm{g}}$ distributions in $\mathrm{Pb}-\mathrm{Pb}$ collisions relative to pp collisions is observed. These measurements are compared to a variety of jet quenching models [33, 35, 37-39, 46-51]. All models considered are consistent with the $z_{\mathrm{g}}$ measurements. Many of the models capture the narrowing effect observed in the $\theta_{\mathrm{g}}$ distributions, although with quantitative differences. This behavior is consistent with models implementing an incoherent interaction of the jet shower constituents with the medium, but also consistent with mediummodified "quark/gluon" fractions with fully coherent energy loss. By isolating the theoretically well-controlled hard substructure of jets, these measurements provide direct connection to specific jet quenching physics mechanisms, and offer the opportunity for future measurements to definitively disentangle them. 

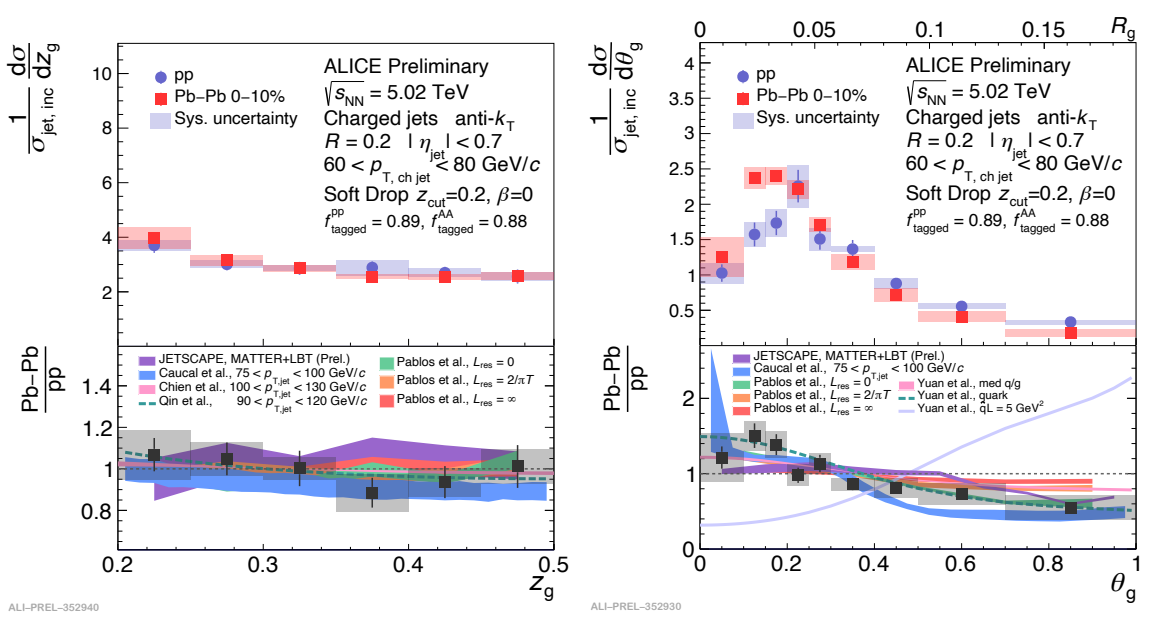

Figure 4: Measurements of $z_{\mathrm{g}}$ (left) and $\theta_{\mathrm{g}}$ (right) in $0-10 \%$ central $\mathrm{Pb}-\mathrm{Pb}$ collisions compared to pp collisions for $R=0.2$, along with comparison to several theoretical models [45].

\section{References}

[1] ALICE Collaboration, PRC 101 (2020), 034911.

[2] V. Khachatryan et al. (CMS Collaboration), EPJ C 76 (2016), 451.

[3] M. Dasgupta, F. A. Dreyer, G. P. Salam and G. Soyez, JHEP 2016 (2016), 57.

[4] Z.-B. Kang, F. Ringer and I. Vitev, JHEP 2016 (2016), 125.

[5] X. Liu, S.-O. Moch and F. Ringer, PRD 97 (2018), 056026.

[6] J. Currie, E. W. N. Glover and J. Pires, PRL 118 (2017), 072002.

[7] M. Czakon, A. van Hameren, A. Mitov and R. Poncelet, JHEP 2019 (2019), 262.

[8] S. Alioli, K. Hamilton, P. Nason, C. Oleari and E. Re, JHEP 2011 (2011), 81.

[9] T. Sjostrand and et al., CPC. 191 (2015), 159.

[10] A. J. Larkoski, I. Moult and B. Nachman, Physics Reports 841 (2020), 1-63.

[11] A. J. Larkoski, S. Marzani, G. Soyez and J. Thaler, JHEP 05 (2014), 146.

[12] Y. Mehtar-Tani, A. Soto-Ontoso and K. Tywoniuk, PRD 101 (2020), 034004.

13] Z.-B. Kang, K. Lee, X. Liu, D. Neill and F. Ringer, JHEP 2020 (2020), 54

[14] ATLAS Collaboration, PRD 101 (2020), 052007.

[15] CMS Collaboration, PRD 98 (2018), 092014.

[16] STAR Collaboration, arXiv: 2003.02114.

[17] Y. Mehtar-Tani, A. Soto-Ontoso and K. Tywoniuk, arXiv: 2005. 07584.

[18] Y. Dokshitzer, G. Leder, S. Moretti and B. Webber, JHEP 1997 (1997), 001.

[19] F. A. Dreyer, G. P. Salam and G. Soyez, JHEP 2018 (2018), 64.

[20] A. J. Larkoski, J. Thaler and W. J. Waalewijn, JHEP 2014 (2014), 129.

[21] ATLAS Collaboration, PRD 86 (2012), 072006.

[22] ALICE Collaboration, JHEP 2018 (2018), 139

[23] Z.-B. Kang, K. Lee and F. Ringer, JHEP 2018 (2018), 110.

[24] Z.-B. Kang, K. Lee, X. Liu and F. Ringer, PLB 793 (2019), 41

[25] J.-W. Qiu, F. Ringer, N. Sato and P. Zurita, PRL 122 (2019), 252301

[26] H. Bossi, 2009.02269.

[27] J. Norman, 2009.08261.

[28] ALICE Collaboration, JHEP 2015 (2015), 170

[29] STAR Collaboration, PRC 96 (2017), 024905.

[30] F. D'Eramo, K. Rajagopal and Y. Yin, JHEP 2019 (2019), 172

31] M. Gyulassy, P. Levai, J. Liao, S. Shi, F. Yuan and X. Wang, NPA 982 (2019), 627-630.

[32] B. G. Zakharov, 2003.10182

33] Y.-T. Chien and I. Vitev, PRL 119 (2017), 112301

[34] Y. Mehtar-Tani and K. Tywoniuk, JHEP 2017 (2017), 125.

[35] N.-B. Chang, S. Cao and G.-Y. Qin, PLB 781 (2018), 423-432.

[36] R. Kunnawalkam Elayavalli and K. C. Zapp, JHEP 7 (2017), 141.

[37] P. Caucal, E. Iancu and G. Soyez, JHEP 10 (2019), 273

[38] F. Ringer, B.-W. Xiao and F. Yuan, PLB 808 (2020), 135634.

[39] J. Casalderrey-Solana, G. Milhano, D. Pablos and K. Rajagopal, JHEP 01 (2020), 044.

[40] H. A. Andrews and et al., JPG 47 (2020), 065102.

[41] CMS Collaboration, PRL 120 (2018), 142302.

[42] ALICE Collaboration, PLB 802 (2020), 135227.

[43] CMS Collaboration, JHEP 2018 (2018), 161.

[44] J. Mulligan and M. Ploskon, arXiv : 2006.01812.

[45] ALICE Collaboration, ALICE-PUBLIC-2020-006, https://cds.cern.ch/record/2725572.

[46] JETSCAPE Collaboration, arXiv: 1903.07706

[47] Y. He, T. Luo, X.-N. Wang and Y. Zhu, PRC 91 (2015), 054908.

[48] A. Majumder, PRC 88 (2013), 014909.

49] P. Caucal, E. Iancu, A. Mueller and G. Soyez, PRL 120 (2018), 232001.

[50] J. Casalderrey-Solana, D. C. Gulhan, J. G. Milhano, D. Pablos and K. Rajagopal, JHEP 019 (2014), 019.

[51] Z. Hulcher, D. Pablos and K. Rajagopal, JHEP 010 (2018), 10. 